\title{
ON STABILITY OF NONLINEAR SYSTEMS AND APPLICATION TO APM MODELING
}

\author{
Jadlovska, A.; Katalinic, B.; Hrubina, K. \& Wessely, E.
}

Abstract: The chapter deals with the investigation of nonlinear systems stability, a characteristic exponent and asymptotic stability. It also deals with the Lyapunov transformation to carry out a linear system whose matrix elements are functions of a system with a constant matrix. The examination of different methods for the construction of Lyapunov functions of nonlinear systems. The stability of systems with changeable parameters as well as the application of nonlinear systems control theory to the problems of "artificial pneumatic muscle - APM" control have also been investigated in the chapter.

Key words: cybernetics, nonlinear systems stability, the Lyapunov transformation, artificial pneumatic muscle
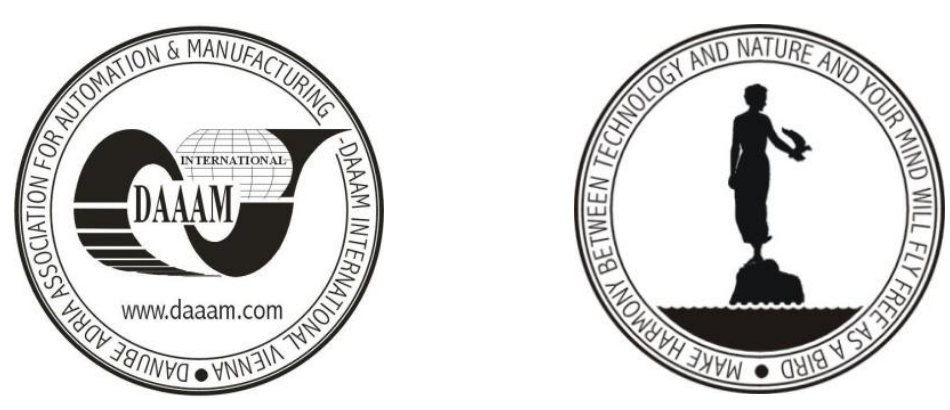

Authors' data: Assoc. Prof. PhD. Jadlovska, A[nna]*, Univ. Prof. Dipl.-Ing. Dr.h.c.mult. Dr.techn. Katalinic, B[ranko]**, Assoc. Prof. PhD. Hrubina, K[amil]***; Assoc. Prof. CSc. Wessely, E[mil]****, *Technical University of Kosice, Letna 1, Kosice, Slovakia,** University of Technology, Karlsplatz 13, 1040, Vienna, Austria, ***Informatech Ltd., Košice, Slovakia, ****University of Security Management in Kosice, Slovakia, anna.jadlovska@tuke.sk, katalinic@mail.ift.tuwien.ac.at, kamil.hrubina@tuke.sk, emil.wessely@vsbm.sk

This Publication has to be referred as: Jadlovska, A[nna]; Katalinic, $\mathrm{B}[\mathrm{ranko}]$; Hrubina, K[amil], \& Wessely, E[mil] (2013) On Stability of Nonlinear Systems and Application to Apm Modeling, Chapter 11 in DAAAM International Scientific Book 2013, pp. 257-276, B. Katalinic \& Z. Tekic (Eds.), Published by DAAAM International, ISBN 978-3-901509-94-0, ISSN 1726-9687, Vienna, Austria DOI: $10.2507 /$ daaam.scibook.2013.11 


\section{Introduction}

The stability of a given system is often defined in the sense that the system is capable of returning to an equilibrium if a signal acting, which led the system out of this state, finished. This definition is sufficient for a linear system, its stability, however, can be defined in a different way, e.g. a linear system is stable if and only if its response to an arbitrary bounded input is bounded. There are several definitions of a nonlinear system stability. Many of them have a limited utilization and were defined for specific cases. In general, the processes going on in linear and nonlinear systems can be expressed by a mathematical model, which actually is a system of differential equations. Lyapunov stability theory enables to investigate the system stability without the necessity of solving either differential equations of the given order or a system of differential equations. A. M. Lyapunov proposed two methods in order to investigate the stability. Lyapunov first method enables to consider the nonlinear system stability according to an approximate linear model, (local stability). Lyapunov second method enables to consider the stability or the asymptotic stability in a certain area $\Omega$, in general with the linear or nonlinear system, (of both excited and unexcited system). When solving the stability problem, the success of the method lies with the investigator'stability to find a suitable function (the so called Lyapunov function)as well as to determine its definiteness, (Athans \& Falb, 1966, Lyapunov, 1950, Jadlovská et al., 2011, Hrubina \& Jadlovská, 2002, 2005, Hrubina,2008).

This chapter will deal with the investigation of nonlinear systems stability described by a vector differential equation, a characteristic exponent and an asymptotic stability. It will also deal with the Lyapunov transformation as well as the stability of the systems with variable coefficients of the system of differential equations. The examination of different methods for the construction of Lyapunov functions of nonlinear systems with the demonstration on the selected problems as well as the application of acquired knowledge to the modeling and control of a defined nonlinear system are also included.

\section{System Stability and Characteristic Exponent}

We will consider a homogeneous linear vector differential equation (or a homogeneous linear system of differential equations)in the form

$$
\dot{\boldsymbol{x}}(\boldsymbol{t})=\boldsymbol{A}(t) \cdot \boldsymbol{X}(t)
$$

where matrix individual elements

$$
\boldsymbol{A}(t)=\left(a_{i j}(t)\right)
$$

are continuous functions in the interval $(a,+\infty)$. For the presentation of the Lyapunov lemma on characteristic exponents and for its deeper understanding, we will use two integral inequalities. 
Lemma 1. (R.Bellman, T.H.Gronwald). Let $f(t)$ and $g(t)$ be nonnegative continuous functions on the interval $\left\langle t_{0},+\infty\right\rangle$, and let $\mathrm{c}$ be a nonnegative constant. Let for $t \geq t_{0}$ be the following inequality valid

$$
f(t) \leq c+\int_{t_{0}}^{t} f(\tau) \cdot g(\tau) d \tau
$$

then also for $t \geq t_{0}$ it holds

$$
f(t) \leq c . \exp \left(\int_{t_{0}}^{t} g(\tau) \cdot d \tau\right)
$$

Proof. The main idea of the proof is that at first we assume that $c>0$, whereas the right-hand side of (3)is also positive and follows of this inequality ( for $t \geq t_{0}$ )thence, by the integration within the limits $t_{0}, t$, we will obtian the inequality

$$
\ln \left[c+\int_{t_{0}}^{t} f(\tau) \cdot g(\tau) d \tau\right]-\ln c \leq \int_{t_{0}}^{t} g(\tau) d \tau
$$

which is the equivalent to the inequality (4)which is being proved.

Let $c=0$. If in this case the right side of (3)is equal to zero, then $f(t)=0$ and it holds (4). Obviously, we also have to consider such existing $t$, for which it holds

$$
\int_{t_{0}}^{t} f(\tau) \cdot g(\tau) d \tau \neq 0
$$

In the process of proving, we will find out that the relation (5)wil be equal ti zero, which is a contradiction, since (5)differs from zero.

Lemma 2. ( R. Bellman, T. H. Gronwald ). Let $f(t)$ be a positive function and $g(t)$ be a nonnegative function on the interval $(a, b)$, and let both functions on this intervale are continuous, and let for the arbitrary $t_{1}, t_{2} \in(a, b)$ it holds

$$
f\left(t_{2}\right) \leq f\left(t_{1}\right)+\left|\int_{t_{1}}^{t_{2}} f(\tau) \cdot g(\tau) d \tau\right|
$$

Then for $a<t_{0} \leq t<b$ the following inequality is satisfied

$$
f\left(t_{0}\right) \leq \exp \left[-\int_{t_{0}}^{t} g(\tau) \cdot d \tau\right] \leq f(t) \leq f\left(t_{0}\right) \cdot \exp \left[\int_{t_{0}}^{t} g(\tau) \cdot d \tau\right]
$$


Further on, we will deal with the investigation of stability of the systems described by the differential equation (1). In doing so, the characteristic exponents will play a similar role to the roots of the characteristic equation for the system of differential equations with constant coefficients. At first we will mention two theorems on stability which do not make use of the notion of a characteristic exponent.s

Theorem 1. A linear system described by the equation (1)is stable in the sense of Lyapunov in the interval $\left\langle t_{0},+\infty\right\rangle$, if all the solution to the equation (1)are bounded functions in the interval $\left\langle t_{0},+\infty\right\rangle$.

Theorem 2. (R. Bellman ). Let all the solutions to the vector differential equation

$$
\dot{\boldsymbol{x}}(t)=\mathrm{A} \boldsymbol{x}(t)
$$

with a constant matrix of $(n, n)$ type be stable in the sense of Lyapunov, or let all the roots of the equation $\operatorname{det} \boldsymbol{A}=0$ have negative real parts. Let $\boldsymbol{B}(\mathrm{t})$ matrix of $(n, n)$ type, where its elements are continuous functions in the interval $\left\langle t_{0},+\infty\right\rangle$ and let the integral be expressed in the form

$$
\int_{t_{0}}^{\infty}\|\boldsymbol{B}(\tau)\| \cdot d \tau<0
$$

Then all the solutions to the equation

$$
\dot{\boldsymbol{y}}(t)=[\boldsymbol{A}+\boldsymbol{B}(\tau)] \cdot \boldsymbol{y}(t)
$$

are stable in the interval $\left\langle t_{0},+\infty\right\rangle$ in the sense of Lyapunov.

\subsection{Characteristic Exponent}

First, we will present stability conditions of linear systems with variable coefficients. The basic notion is that of a characteristic exponent of the function introduced by A.M. Lyapunov.

Definition 1. A characteristic exponent of a complex function $f(t)$ of a real variable $t$ is called a number.

$$
\chi(f)=\limsup _{t \rightarrow \infty}(\mathrm{t})^{-1} \ln |f(\mathrm{t})|
$$

In order to explain this notion by which a growth velocity of a function is characterized, it is sufficient to realize the following fact. A module of the given function can be expressed in the form 


$$
|f(t)|=e^{\alpha(t), t}
$$

whereas

$$
\alpha(t)=(t)^{-1} \cdot \ln |f(t)|
$$

Asymptotic behaviour of this function $\alpha(t)$ for $t \rightarrow \infty$ is expressed by the relation (11). Obviously for the real $\alpha$ it holds

$$
\chi\left(e^{\alpha t}\right)=\alpha
$$

It is possible to derive many basic properties of a characteristic exponent.

Lemma 3. Let for $m>0$ and for $t>t_{o}$ be

$$
|f(t)| \leq t^{m}
$$

then $\quad \chi(f(t))=0$

The assertion is a direct consequence of the relation (11).

Lemma 4. Let $\varphi(t)$ be a complex function defined for all $t>t_{o}$ and bounded $|\varphi(t)| \leq c$

then $\quad \chi\left(e^{t . \varphi(t)}\right) \leq c$

Lemma 5. Let for $t>t_{o}$ be defined the functions $f(t), g(t)$ ans let for this $t$ them satisfy the inequality

$$
|f(t)| \leq|g(t)|
$$

then there is $\quad \chi(f)=\chi(g)$

The assertion follows from the relation (11)since the logarithm is an increasing function of a real variable.

Lemma 6. Let $f(t)$ be defined for $t>t_{o}$ and let its characteristic exponent be finite $\chi(f)=\alpha$

then for each $\varepsilon$ the following relations are valid

$$
\begin{aligned}
& \lim _{t \rightarrow \infty}|f(t)|\left[e^{(\alpha+\varepsilon) \cdot t}\right]^{-1}=0 \\
& \lim _{t \rightarrow \infty} \mid f(t)\left[e^{(\alpha-\varepsilon) \cdot t}\right]^{-1}=+\infty
\end{aligned}
$$

If, on the contraty, for some $\alpha$ the equality (14)is valid for the function $f(t)$ then

$$
\chi(f) \leq \alpha
$$


If the equality (15)is valid, then

$$
\chi(f) \geq \alpha
$$

Lemma 7. For the characteristic exponent of the sum of a finite number of the functions $f_{k}(t)$ definit for $t>t_{o}$, the following inequality holds

$$
\chi\left(\sum_{k=1}^{n} f_{k}\right) \leq \max _{1 \leq k \leq n} \chi\left(f_{k}\right)
$$

If all characteristic exponents are finite and if there exists the index $p$, $1 \leq p \leq n$, such that

$$
\chi\left(f_{p}\right)>\chi\left(f_{k}\right), k \neq p, 1 \leq k \leq n,
$$

then

$$
\chi\left(\sum_{k=1}^{n} f_{k}\right)=\chi\left(f_{p}\right)
$$

Lemma 8. Let the function $f_{k}(t)$ and $c_{k}(t), k=1,2, \ldots, n$ be definit for $t>t_{o}$

and let the function $c_{k}(t)$ be bounded. Then for the characteristic exponent of a linear combination the following inequality is valid

$$
\chi\left(\sum_{k=1}^{n} c_{k} f_{k}\right) \leq \max _{1 \leq k \leq n} \chi\left(f_{k}\right)
$$

Definition 2. Let the function $f(t)$ be defined and let for $t \geq t_{0}$ there exists a definite integral, a primitive function to the function $f(t)$ in terms of Lyapunov will be called the function $F(t)$ given by the rule

$$
\begin{aligned}
& F(t)=\int_{t_{0}}^{t} f(\tau) d \tau \text { for } \chi(f) \geq 0 \\
& F(t)=\int_{t}^{+\infty} f(\tau) d \tau \text { for } \chi(f)<0
\end{aligned}
$$

The characteristic exponent of the primitive function in terms of Lyapunov is not higher than that of a related subintegral function. The characteristic exponent of the function $F(t)$ matrix is equal to the characteristic exponent of its norm, i.e. for an arbitrary selected norm. 
Theorem 3. ( A. M. Ljapunov ). If the matrix (2)in the equation (1)is norm bounded ( we can assume an arbitraty norm ).

$$
\|\boldsymbol{A}(t)\| \leq C<+\infty,
$$

Then every non-zero solution $\boldsymbol{x}(t)$ has the infinite characteristic exponent.

Now we can carry out a closer investigation of the set of characteristic exponents of the solution to the linear system described by the equation (1)with a bounded matrix (2).

Lemma 9. Non-zero vector functions $x_{i}(t), i=1,2, \ldots, m$, definited on the interval $\left\langle t_{0},+\infty\right\rangle$ and having mutually different characteristic exponents are linearly independent. The proof of the lemma can be done by a contradiction.

Definition 3. A set of all finite characteristic exponents of the solutions to the system of differential equations (nonlinear in general)is called a spectrum of this system. First, let us assume the system of first order linear differential equations a matrix, whose elements are constants (1). In general, each component of the solution to this system can be expressed in the form of a linear combination

$$
x_{j}(t)=\sum_{i=1}^{m} c_{i j} P_{i}(t) e^{\lambda_{i} t}
$$

where $P_{i}(t)$ are the polynomials in $t$ and $\lambda_{i}$ are eigennumbers of the matrix $\boldsymbol{A}$ the roots of the equation

$$
\operatorname{det}(\boldsymbol{A}-\lambda E)=0
$$

thus it is

$$
\chi\left(P(t) e^{\lambda t}\right)=R_{e} \lambda
$$

and a characteristic exponent of the solution $x(t)$ is thus equal to a real part of some of the matrix $\boldsymbol{A}$ eigennumbers. In the sense of the definition 2. Spectrum is identical to the sets of real parts of the matrix $\boldsymbol{A}$. The case in which the $\boldsymbol{A}$ matrix in (2)has changeable elements will be described in the following theorem.

Theorem 4. Spectrum of the system of linear homogenous differential equations (1)of the $m$-th order is a finite set of numbers

$$
\alpha_{1}<\alpha_{1}<\alpha_{1}<\ldots<\alpha_{m} \text {, where } m \leq n
$$

Necessary and sufficient conditions for asymptotic stability of the solution to the system with a matrix whose elements are constant is that the roots of the characteristic equation have negative real parts. Similarly, for the system with a 
Jadlovska, A.; Katalinic, B.; Hrubina, K. \& Wessely, E.: On Stability of Nonlinear ... matrix whose elements are functions of the parameter, we will show that the characteristic exponent is negative, (Lyapunov, 1950, Barbašin \& Krasovskij, 1954).

\subsection{Asymptotic Stability of a Vector Differential Equation and a Charateristic Exponent}

Theorem 5. In the sense of Lyapunov, for the asymptotic stability of a linear homogeneous system described by the vector equation (1)it is sufficient that its maximum characteristic exponent is negative.

Using the characteristic exponents, it is possible to characterize a set of solutions to the linear homogeneous system as follows, let

$$
\left\{\boldsymbol{X}_{1}(t), \boldsymbol{X}_{2}(t), \ldots ., \boldsymbol{X}_{n}(t)\right\}
$$

be a fundamental system of solutions to the equation (1)and let

$$
\chi\left(\boldsymbol{x}_{j}\right)=\alpha_{j}, \quad j=1,2, \ldots . ., n
$$

We remark that the numbers (21)are not necessarily mutually different.

Let us denote

$$
\boldsymbol{X}_{j}(t)=e^{\alpha_{j}(t)} \cdot \xi_{j}(t)
$$

From the above, it follows that

$$
\chi\left(\xi_{j}\right)=0
$$

In general, the solution to the system (1)has the form

$$
\boldsymbol{X}(t)=\sum_{j=1}^{n} c_{j} \xi_{j}(t) e^{\alpha_{j} t}
$$

where $c_{j}$ are the constants, $\xi_{j}(t) e^{\alpha_{j} t}$ are linear independent solutions (20), $\alpha_{j}$ are the elements for the matrix (2)spectrum and $\xi_{j}(t)$ have the property (23).

Let all the numbers (21)be finite and let the $n$-tuple (21)be created by mutually different numbers $\alpha_{k}, k=1,2, \ldots ., m$, where, certainly, $m \leq n$. Let $v_{k}$ be a number of solutions (20), which have a characteristic exponent $\alpha_{k}$. In dependence on a selected fundamental matrix (20), let us denote it $(\boldsymbol{X})$, we can construct a number

$$
s(\boldsymbol{X})=\sum_{k=1}^{m} v_{k} \alpha_{k}
$$


Fundamental systems with a minimum number $s(\boldsymbol{X})$ are sometines called normal. According to Lyapunov, it is possible to derive a low estimation of a number (25).

Lemma 10. Let there be given the system (1)whose spectrum (19)contains only finite numbers and let $\boldsymbol{X}(t)$ be its fundamental matrix. Then the number (25)satisfies the inequality

$$
s(\boldsymbol{X}) \geq \lim _{t \rightarrow+\infty} \sup \frac{1}{t-t_{0}} \int_{t_{0}}^{t} s p \boldsymbol{A}(\tau) d \tau
$$

Where the symbol $\operatorname{sp} \boldsymbol{A}(\tau)$ is used to denote the matrix $\boldsymbol{A}$ trace, i.e. the sum of the elements on the main diagonal.

\section{Lyapunov Transformation}

When investigating the stability of solutions to homogeneous linear systems (1) in some cases it is possible to find a linear transformation

$$
\boldsymbol{y}(t)=\boldsymbol{L}(t) \cdot \boldsymbol{X}(t)
$$

which will change a system (1)with $\boldsymbol{A}(t)$ matrix to the system

$$
\dot{y}(t)=B y(t)
$$

with a constant matrix. If during this transformation the characteristic exponents are not changed, it is possible to solve the problem of stability of the system (1)by means of known methods.

Definition 4. Let matrix $\boldsymbol{L}(t)$, whose elements have continuous first derivatives in the interval $\left.<t_{0},+\infty\right)$ is called Lyapunov matrix, if

a) $\sup \|\boldsymbol{L}(t)\| \quad$ and $\left.\sup \|\dot{\boldsymbol{L}}(t)\| \quad, t \in<t_{0},+\infty\right)$; are finite numbers

b) $\left.\quad\|\operatorname{det} L(t)\| \geq k>.0 \quad \forall t \in<t_{0},+\infty\right)$

The corresponding transformation (27)is called Lyapunov transformation.

Lemma 11. The transformation(27), in which the matrix $\boldsymbol{L}(t)$ is the Lyapunov matrix in terms of the definition 4 , does not change the characteristic exponent, i.e.

$$
x y)=\chi(x)
$$

The important result related to the issue of transformability to a system with a constant matrix while preserving the spectrum was published by N.P.Yurigin. The following lemma is valid: 
Theorem 6 . A linear system described by the equation (1), can be transformed by the Lyapunov transformation with the matrix $\boldsymbol{L}(t)$ to the system with a constant matrix $\boldsymbol{B}$ if at least one its fundamental matrix $\boldsymbol{X}(t)$ can be expressed in the form

$$
X(t)=L(t) \exp (\boldsymbol{B} . t)
$$

Let us mention another different method of transformation of the problem refferend to asymptotic stability of a system with a time-varying matrix to the problem of stability of the system with a constant matrix. For this purpose, we first will define the following:

Definition 5. The two systems described by vector differential equations

$$
\dot{\boldsymbol{x}}_{i}=f_{i}\left(t, \boldsymbol{x}_{i}\right), \quad i=1,2
$$

are called asymptotically equivalent when each solution $\boldsymbol{x}_{k}$ of any of both systems corresponds with the solution $\boldsymbol{X}_{l}$ of the second system, thus

$$
\lim _{t \rightarrow \infty}\left(\boldsymbol{X}_{k}(t)-\boldsymbol{X}_{l}(t)\right)=0
$$

A simple criterion of asymptotic equivalence for the systems with o constant matrix was created in 1946 by an American mathematician N. Levinson. His Lemma is used here without a proof because it is rather lengthy, (Codington \& Levinson, 1955).

Lemma 12. If all solutions of the system (28)with a constant matrix are bounded on the interval $\left\langle t_{0},+\infty\right\rangle$ and if the matrix $\boldsymbol{C}(t)$ whose elements are continuous functions on the interval $\left\langle t_{0},+\infty\right\rangle$ satisfies the inequality

$$
\int_{t_{0}}^{+\infty}\|\boldsymbol{C}(\tau)\| d \tau<+\infty
$$

then the system $\dot{\boldsymbol{x}}(t)=(\boldsymbol{B}+\boldsymbol{C}(t)) \boldsymbol{X}(t)$

is asymptotically equivalent to the system (28).

An interesting consequence of Levinson's lemma is the asymptotic behaviour of the solution to the system

$$
\dot{\boldsymbol{X}}(t)=\boldsymbol{C}(t) \boldsymbol{X}(t)
$$

where the matrix $\boldsymbol{C}(t)$ satisfies the inequality (32). This system is in fact asymptotically equivalent to the system 


$$
\dot{y}(t)=0
$$

and each component of the vector $\boldsymbol{x}(t)$ of the system (34)converges for $t \rightarrow+\infty$ to some constant, on the contrary, for an arbitrary constant vector $c \in R^{n}$ there exists such solution $\boldsymbol{X}(t)$ to the system (34)that

$$
\lim _{t \rightarrow+\infty} \boldsymbol{X}(t)=\boldsymbol{C} .
$$

\section{Stability of Systems with Variable Coefficients}

Differential equations which are used to describe the systems with variable parameters, have time-varying coefficients; they will be denoted $a_{i}(t)$ time functions. The stability of the systems with variable parameters can be secured only in a certain time interval. Beyond this interval, the system can be instable, (Jadlovská et al.2011).

\subsection{Basic Relation}

We will investigate the system with variable parameters described by the differential equation

$$
a_{n}(t) x_{2}^{(n)}(t)+\ldots . .+a_{1}(t) x_{2}^{\prime}(t)+a_{0}(t) x_{2}(t)=u(t)
$$

$x_{2}(t)$ is an output value, $u(t)$ is an input value. Our task is to find a relation between the input and the output values of the investigated system for such a case that the system is in an equilibrium until the moment when the input signal starts acting. The solution is considered from the moment when the input signal is applied. For this moment it holds:

$$
\left.x_{2}^{(v)}(t)\right|_{t=0}=0, \quad v=1,2, \ldots .,(n-1)
$$

The solution to the equation (36)will be obtainedby the variation of constants methods. The considered solution is searched for in the form

$$
x_{2}(t)=\varphi_{1}(t) \gamma_{1}(t)+\varphi_{2}(t) \gamma_{2}(t)+\ldots+\varphi_{n}(t) \gamma_{n}(t)
$$

where $\varphi_{i}(t)$ are linear independent particular solutions to the homogeneous equation, $\gamma_{j}(t)$ will be determined in such a way that after inserting the expression (38)into (36)we obtaint the identity.

Thus we obtain the solution in the form:

$$
x_{2}(t)=\int_{0}^{t} g(t, v) u(v) d v
$$

In order to explain the physical substance of the , $g(t, v)$, function, we will investigate the case in which at the moment $t=\xi$ for the system input there is introduced a signal in the form of the Dirac impulse, i.e. 


$$
u(v)=\delta(v-\xi), \quad 0<\xi<v
$$

If we apply the exppresion (39)and the known equality

$$
\int_{-\infty}^{\infty} f(t) \cdot \delta(t-\xi) d t=f(\xi)
$$

Thus we obtain an impulse transition function of the system, which is described by the equation (38)

$$
\int_{0}^{t} g(t, v) \cdot \delta(t-\xi) d v=g(t, \xi)
$$

The impulse transition function is called the system response ( which before the beginning of the signal acting was in an equilibrium)to the input signal in the form og the Dirac impulse. Considering a mathematical point of view, $g(t, \xi)$ is the solution to the differential equation

$$
a_{n}(t) g^{(n)}(t, \xi)+\ldots . .+a_{1}(t) g^{\prime}(t, \xi)+a_{0}(t) g(t, \xi)=\delta(t-\xi)
$$

with the initial conditions

$$
\left.g^{(v)}(t, \xi)\right|_{t=\xi}=0, \quad v=0,1,2, \ldots,(n-1)
$$

The impulse transition function can also be applied to a more general case for the systems with changeable parameters to solve the equation in the form:

$$
\begin{aligned}
& a_{n}(t) w^{(n)}(t, \xi)+\ldots . .+a_{1}(t) w^{\prime}(t, \xi)+a_{0}(t) w(t, \xi)= \\
& =b_{m}(t) \delta_{m}^{(m)}(t-\xi)+\ldots . .+b_{1}(t) \delta_{1}^{\prime}(t-\xi)+b_{0}(t) \delta_{0}(t-\xi)
\end{aligned}
$$

with the initial conditions

$$
\left.w^{(v)}(t, \xi)\right|_{t=\xi}=0, \quad v=0,1,2, \ldots,(n-1)
$$

In this case, $w(t, \xi)$ represents an impulse transition function of the systems with the changeable parameters of a general form. The $w(t, \xi)$ function is related to the $g(t, \xi)$ function according to the relation

$$
w(t, \xi)=(-1)^{m} \frac{d^{m}}{d \xi^{m}}\left[g(t, \xi) b_{m}(\xi)\right]+\ldots+g(t, \xi) b_{0}(\xi)
$$




\subsection{Determination of a Parametric Transfer Function}

The analysis of systems with variable parameters almost never utilises direct relationships between input and output variables, but the Laplace and Fourier transforms are used instead. These transforms are used to simplify the calculation and perform a simple transfer to the frequency domain. The use of the mentioned transforms for the analysis of the systems with variable parameters is of a great practical importance and extends the possibilities of the analytical investigation of automatic control systems. Significant results in this field were achieved by (L.A.Zadech, (1953, Hrubina \& Jadlovská, 2005, Jadlovská et al.2005).

Let us assume that for the input signal of the system with variable parameters there exists Fourier integral

$$
x_{1}(t)=\frac{1}{2 \pi} \int_{-\infty}^{\infty} X_{1}(j \omega) e^{j \omega t} d v
$$

where $X(j \omega)$ is a complex relative amplitude of the $x_{1}(t)$ function spectrum.

Instead of the function $x_{1}(t)$ connected to the input of the system at the moment $t$ $=0$, there will operate now an ,infinite number" of sinusoidal oscillations starting at

$t=-\infty$. In the relation for the output variable, this factor has to be respected by moving the lower limit of integration

$$
x_{2}(t)=\int_{-\infty}^{t} w(t, \xi) x_{1}(\xi) d \xi
$$

If we substitute the expression (43)to the equality (44)and we replace the order of integration, after a simple modification we obtain

$$
x_{2}(t)=\frac{1}{2 \pi} \int_{-\infty}^{\infty} X_{1}(j \omega) e^{j \omega t}\left[\int_{-\infty}^{\infty} w(t, \xi) e^{-j \omega(t-\xi)} d \xi\right] \cdot d \omega
$$

If we introduce the denotation

$$
W(j \omega, t)=\int_{-\infty}^{t} w(t, \xi) e^{-j \omega(t-\xi)} \cdot d \xi
$$

We can write

$$
x_{2}(t)=\frac{1}{2 \pi} \int_{-\infty}^{\infty} W(j \omega, t) X_{i}(j \omega) e^{j \omega t} \cdot d \omega
$$

If we use the known methods to convert the Fourier transform to the Laplace transform, we can write the following expressions

$$
x_{2}(t)=\frac{1}{2 \pi j} \cdot \int_{c-j \omega}^{c+j \omega} W(s, t) \boldsymbol{X}_{1}(s) e^{s t} . d s
$$




$$
W(s, t)=\int_{-\infty}^{s} W(t, \xi) e^{-s(t-\xi)} \cdot d \xi
$$

(Poznyak, 2008, Tripathi, 2008, Hrubina \& Jadlovská, 2005).

The expression (45)is a more general expression of the known expression for the transfer of the system with constant parameters. They differ only in the parameter $t$, which occurs in the function $W(s, t)$. In connection with this fact, the function $W(s$, $t$ )will be called a parametric transfer function of the system. The searched output value is simply determined from the relation

$$
X_{2}(s, t)=W(s, t) X_{1}(s)
$$

The relation (45)is primarily used to determine the parametric transfer function which can be used for the stability control of the system with variable parameters.

\section{Methodology for Constructing the Lyapunov Function}

For nonlinear differential equations of a lower order we can find a simple physical interpretation of the Lyapunov theory and based on it desing a suitable Lyapunov function $V(x)$. This method is often used in theoretical mechanics, robotics, etc.; with its help we can clarify the relations between the Lyapunov stability theory and some of the theories of optimal systems, especially the Pontryagin maximum principle and dynamic programming, (Hrubina, 2008).

\subsection{Constructing the Lyapunov Function based on the Physical Analogy}

Let us consider a simple mechanical system spring- mass- damper, in which a directive force $f(x)$ and a damping force $g(\dot{x})$ are nonlinear. The motion of the system can be expressed by the differential equation

$$
\ddot{x}+g(\dot{x})+f(x)=0 \quad x(0)=x_{0}, \quad \dot{x}(0)=\dot{x}_{0}
$$

With the conservative system, the damping is $g(\dot{x})=0$ and the total energy is constant. If this system is solved in the phase level with the coordinate $x_{1}=x, x_{2}=\dot{x}, \quad$ then (46)is expressed in the form

$$
\begin{aligned}
& \dot{x}_{1}=x_{2} \\
& \dot{x}_{2}=-f\left(x_{1}\right)
\end{aligned}
$$

For $f\left(x_{1}\right) \neq 0$ at $x_{1} \neq 0$ and $f(0)=0$ the system has one equilibrium in the beginning. The trajectories are closed curves surrounding the beginning and the following relation is valid for them

$$
\frac{d x_{2}}{d x_{1}}=-\frac{f\left(x_{1}\right)}{x_{2}}
$$


By the integration we obtain the trajectory equation

$$
\frac{x_{2}^{2}}{2}+\int_{0}^{x_{1}} f\left(x_{1}\right) d x_{1}=\text { cont }
$$

The first member represents a kinetic energy and the second one represents a potential energy. Their sum is the total energy $E\left(x_{1}, x_{2}\right)$ of a moving system. Thus, the trajectories of the system are the curves are the total energy of the conservative system. The change with time of the total energy is zero.

$$
\dot{E}\left(x_{1}, x_{2}\right)=x_{2} \dot{x}_{2}+f\left(x_{1}\right) \dot{x}_{1}=x_{2}\left[\dot{x}_{2}+f\left(x_{1}\right)\right]=0
$$

\subsection{Constructing the Lyapunov Function of a Nonlinear System by analogy to the Linear System}

Let us consider a nonlinear system described by the differential equation

$$
\ddot{x}+\varphi(x) \dot{x}+f(x)=0
$$

Which corresponds to the system

$$
\begin{gathered}
\dot{x}_{1}=x_{2} \\
\dot{x}_{2}=-f\left(x_{1}\right)-\varphi\left(x_{1}\right) x_{2}
\end{gathered}
$$

Now let us consider the linear system

$$
\begin{gathered}
\dot{x}_{1}=x_{2} \\
\dot{x}_{2}=-f\left(x_{1}\right)-a_{2} \cdot x_{2}
\end{gathered}
$$

For which we will construct the Lyapunov function of the first type, i.e. we will consider such function that its time derivative is negative semidefinite

$$
\dot{V}\left(x_{1}, x_{2}\right)=-a_{2} x_{2}^{2}
$$

Therefore, we will search for the Lyapunov function in a quadratic form:

$$
V\left(x_{1}, x_{2}\right)=k_{11} x_{1}^{2}+2 k_{12} x_{1} x_{2}+k_{22} x_{2}^{2}
$$

Of which by a derivation we will obtain a set of three equations in three unknowns and their values will be : $k_{11}=\frac{a_{1}}{2}, k_{12}=0, k_{22}=1 / 2$, thus 


$$
V\left(x_{1}, x_{2}\right)=\frac{1}{2}\left(a_{1} x_{1}^{2}+x_{2}^{2}\right)
$$

Since this function does not depend on the coefficient ${ }^{a_{2}}$, it can be used for the Lyapunov function of the first type, for example, for the system

$$
\begin{gathered}
\dot{x}_{1}=x_{2} \\
\dot{x}_{2}=-a_{1} x_{1}-\varphi\left(x_{1}\right) \cdot x_{2}
\end{gathered}
$$

In order to construct the Lyapunov function of the first type for the system (49), it is necessary to replace the first member on the right-hand side of the equation (52). The values $a_{1} x_{1}$, eventually $f\left(x_{1}\right)$ represent the force acting on the mass point, which keeps it on the move. The value $\frac{1}{2} a_{1} x_{1}^{2}$ corresponds to the potential energy. Therefore, let us consider the function

$$
V\left(x_{1}, x_{2}\right)=\int_{0}^{x_{1}} f(\xi) d \xi+\frac{1}{2} x_{2}^{2}
$$

which is identical to the function (52)if $f\left(x_{1}\right)=a_{1} x_{1}$. We simply ascertain that

$$
\dot{V}\left(x_{1}, x_{2}\right)=-\varphi\left(x_{1}\right) \cdot x_{2}^{2}
$$

if the condition $\varphi\left(x_{1}\right) \geq 0$, is satisfied and at the same time if for $x \neq 0, \quad f(x) . x \geq 0$ the function $V\left(x_{1}, x_{2}\right)$ is the Ljapunov function of the first type for the given problem because then the right-hand side of the equality (53)is positive definite, (Malkin, 1966).

\subsection{Energy-based Method for the Lyapunov Function Constructing}

Let the given nonlinear system be described by a nonlinear differential equation

$$
\ddot{x}+f(x) \cdot \dot{x}+g(x)=u(t)
$$

where the function $u(t)$ represents an input signal to the system. The differential equation (54)is equivalent to the system of first order differential equations

$$
\begin{aligned}
& \dot{x}_{1}=x_{2} \\
& \dot{x}_{2}=-g\left(x_{1}\right)-f\left(x_{1}\right) \cdot x_{2}+u(t)
\end{aligned}
$$

which can be expressed by one equation

$$
x_{2} d x_{2}=-g\left(x_{1}\right) d x_{1}-f\left(x_{1}\right) x_{2} d x_{1}+u d x_{1}
$$


For the application of the energy-based method, we will denote $E_{s}(x)$ energy accumulated in the system and $E_{1}(x)$ interactive energy, then, after a slight modification, we obtian the equation

$$
g\left(x_{1}\right) d x_{1}+x_{2} d x_{2}=-f\left(x_{1}\right) x_{2} d x_{1}+u d x_{1}
$$

The system under investigation will be srable if, with the increasing time, an interactive energy (the right-hand side of the equation (56))decreases, i.e.

$$
\dot{E}_{1}=-f\left(x_{1}\right) x_{2} \dot{x}_{1}+u \dot{x}_{1}<0
$$

Thus, the system is stable if

$$
-f\left(x_{1}\right) \cdot x_{2}^{2}<0 \text { and } f\left(x_{1}\right)>0 ; \quad \int_{0}^{x_{1}} g(\xi) d \xi>0, g(\xi)>0 \text { at } \xi>0
$$

In the relation (58), the integral expresses a positive value of the system energy.

\section{Control of the APM non Linear System}

From the theoretical point of view, modelling and control of a pneumatic actuator, called "artificial pneumatic muscle" (APM), is a complex problem. The APM control is considerably complicated owing to its simple design, especially because of its nonlinearity, air compressibility, time varying properties as well as the difficulties in the analytical modelling, Fig 1a, b.

In general, APM is investigated from the viewpoint of the theory of nonlinear systems, since the mathematical model is expressed by a second order nonlinear differential equation in the form

$$
M \ddot{\boldsymbol{x}}+B(\dot{\boldsymbol{x}}) \dot{\boldsymbol{x}}+K(\boldsymbol{x}) \boldsymbol{x}=u
$$

or, assuming that a total mass is unity, i.e. $M=1$; and the remaining physical values have a usual meaning, whereas the nonlinear functions are denoted $f_{1}(\dot{\boldsymbol{x}}), f_{2}(\boldsymbol{x})$ , then the equation (59)can be expressed as follows:

$$
\ddot{\boldsymbol{x}}+f_{1}(\dot{\boldsymbol{x}}) \dot{\boldsymbol{x}}+f_{2}(\boldsymbol{x}) \cdot \boldsymbol{x}=u^{*}
$$

This equation is equivalent to the system of the first order differential equations

$$
\begin{array}{lll}
\left(x_{1}=x \quad \text { a } \quad x_{2}=\dot{x}\right) & \dot{x}_{1}=x_{2} \\
& \dot{x}_{2}=u-f_{1}(\dot{x}) \dot{x}-f_{2}(x) x
\end{array}
$$

or in the matrix expression 
Jadlovska, A.; Katalinic, B.; Hrubina, K. \& Wessely, E.: On Stability of Nonlinear ...

$$
\begin{gathered}
\left(\begin{array}{l}
\dot{x}_{1} \\
\dot{x}_{2}
\end{array}\right)=\left(\begin{array}{cc}
0 & 1 \\
-f_{2}\left(x_{1}\right) & -f_{1}\left(x_{2}\right)
\end{array}\right)\left(\begin{array}{l}
x_{1} \\
x_{2}
\end{array}\right)+\left(\begin{array}{l}
0 \\
1
\end{array}\right) u \\
y(t)=x(t)=x_{1}(t)
\end{gathered}
$$

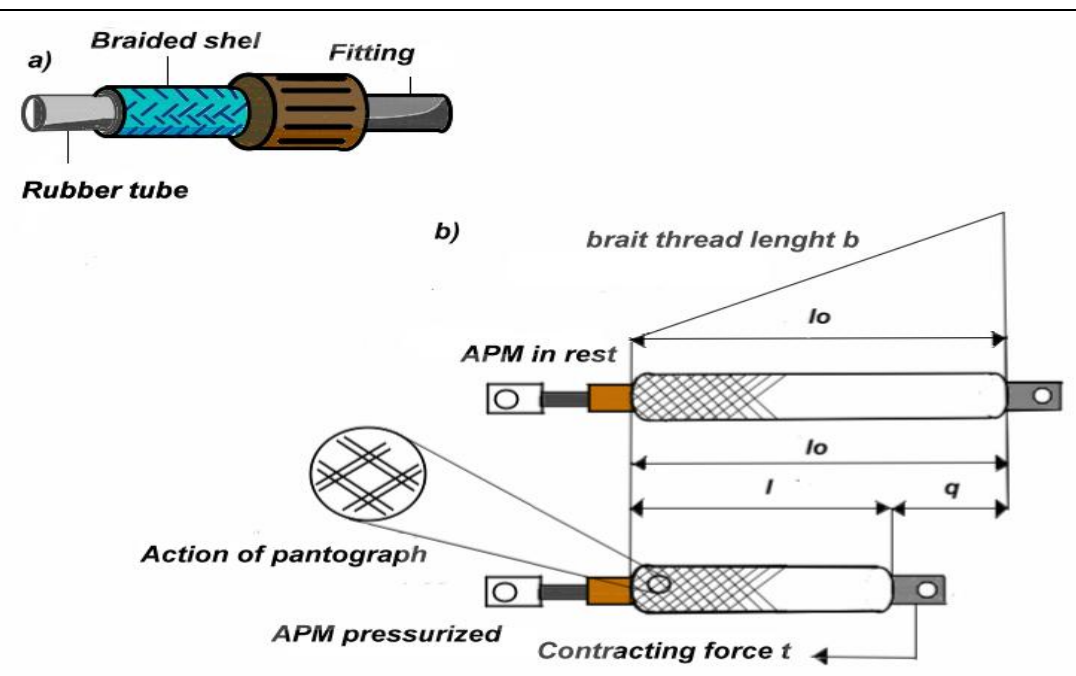

Fig. 1. The basic design of the artificial pneumatic muscle (a), its functions (b)

Further on, we assume that nonlinear functions $f_{1}\left(x_{2}\right), f_{2}\left(x_{1}\right)$ can be expressed by means of second order polynomials, i.e.

$$
\begin{aligned}
& f_{1}\left(x_{2}\right)=B\left(x_{2}\right) x_{2} \\
& f_{2}\left(x_{1}\right)=K\left(x_{1}\right) x_{1}
\end{aligned}
$$

and let us write

$$
\begin{aligned}
& B\left(x_{2}\right)=b_{2} x_{2}^{2}+b_{1} x_{2}+b_{0} \\
& K\left(x_{1}\right)=a_{2} x_{1}^{2}+a_{1} x_{1}+a_{0}
\end{aligned}
$$

The $b_{i}, a_{i}(i=0,1,2)$ coefficients can be obtained by the identification of the measured values

$$
\begin{aligned}
& K\left(x_{1}\right)=K(x) \text { represents a model (inflation and deflation }- \text { hard spring) } \\
& B\left(x_{2}\right)=B(\dot{x}) \text { represents a model (inflation and deflation),(Malkin, 1966). }
\end{aligned}
$$

\section{Lyapunov Function and Law of Control}

If there is a mathematical model (APM)designed, which is represented by a second order nonlinear differential equation, it is possible to express a Lyapunov function and a control: 


$$
\begin{gathered}
M \ddot{\boldsymbol{x}}+f_{1}(\dot{\boldsymbol{x}}) \dot{\boldsymbol{x}}+f_{2}(\boldsymbol{x}) \boldsymbol{x}=u \equiv F \\
V_{2}=\frac{1}{2} M \dot{x}^{2}+\int_{0}^{x} f_{2}(\tau) \tau d \tau \\
\dot{V}_{2}=F \dot{x}-\left[f_{1}(\dot{x}) \dot{x}^{2}\right]
\end{gathered}
$$

Let us denote the $x_{d}(t)$ reference trajectory and let $u(t)$ be clearly known, then the relations (60), (61)are valid. For a feedback after a linearization, it is possible to write a dependence:

$$
u=f_{2}\left(x_{1}\right) x_{1}+f_{1}\left(x_{2}\right) x_{2}+\ddot{x}_{d}-\lambda \dot{x}
$$

Then the system

$$
\begin{aligned}
& \dot{x}_{1}=x_{2} \\
& \dot{x}_{2}=\ddot{x}_{d}-\lambda\left|\dot{x}_{1}-\dot{x}_{d}\right|
\end{aligned}
$$

is stable. Based on this it follows that

$$
\dot{x}_{2}+\lambda x_{2}=\ddot{x}_{d}+\lambda \dot{x}_{d}
$$

By virtue of Lyapunov theory, it is possible to show that the derivatives of the reference trajectory $x_{d}(t)$ are of the exponential order, thus the solutions $x_{1}(t)$ and $x_{2}(t)$ are exponentially stable, (Hrubina, 2008).

\section{Conclusion}

The contribution of the paper consists in the processing of the achieved results based on a considerably wide theoretical part on stability of linear and nonlinear systems expressed by a mathematical model which is represented by a homogeneous linear system of differential equations (a homogeneous linear vector differential equation)with changeable coefficients based on Lyapunov first and second method. In order to solve the problems of stability defined by a linear vector differential equation with the $A(t)$ changeable matrix, Bellman, Gronwald and Lyapunov lemmas and theorems were applied. This refers to the theorems utilizing the defined notion of a characteristic exponent, a matrix spectrum, but especially the Lyapunov transformation.

The essence of the presented methods of solution applied to the problems of asymptotic stability of the system with a time varying matrix lies in the application to the problem of the system stability with a constant matrix. The mentioned possibility of the problem solving is proved by means of the Lyapunov transformation and Levinson theorem. Another contribution lies in the solution of the problems of stability of the systems with changeable parameters which are described by a system 
Jadlovska, A.; Katalinic, B.; Hrubina, K. \& Wessely, E.: On Stability of Nonlinear ...

of differential equations with time-varying coefficients. The paper presents the solution to the problem with the utilization of the variation of constants method and the notion of impulse transition function of the system. The presented theoretical knowledge is applied to the control of the APM nonlinear system.

\section{Acknowledgement}

This work has been supported by the Scientific Grant Agency of Slovak Republic under project Vega No.1/0286/11 Dynamic Hybrid Architecture of the Multiagent Network Control System.

\section{References}

Athans,M.,Falb,P.(1966). Optimal Control (An introduction to the Theory and Its Aplications). p.867, McGRAW - HILL BOOK COMPANY, New York

Barbašin,E., Krasovskij,H.H. (1954). Existence of the Lyapunov Function in Case of Global Symptotic Stability, p. 345-350, PMM 18, 3

Codington, E., Levinson, N., (1955). Theory of ordinary diffential equations. Mc, Graw - Hill, New York

Hrubina, K. and Jadlovská, A. (2002). Optimal Control and Approximation of Variational Inequalities. Kybernetes The International Journal of Systems and Cybernetics, pp.1401-1408, Vol 9/10, Emerald, ISSN 0368-492X

Hrubina, K., Jadlovská, A.(2005)Optimal Control of Process based on the use of Informatics Methods, p.287, Informatech, Ltd. ISBN 80-88941-30-X, Košice

Hrubina, K.,(2008). Stability and development of Lyapunov function of nonlinear systems, p.5-17,In:Modeling, simulation and verify selected Mechatronic systems,(in Slovak). Balara,M. (Ed), Tribun EU Brno, p.136, ISBN 978-80-7399-541-6.

Jadlovská, A., Hrubina,K.,\& Katalinc.,B.,(2005). Algorithm for optimal Decision Making Processes Control, Chapter 21,DAAAM International Scientific Book, In Katalinič, B. (Ed), Vienna, Austria, pp.253-290, ISBN: 3-901509-43-7

Jadlovská, A.(2004)The Dynamic processes Modeling and Control using the Neuron network, p.174, Scientific Book Writing Edition of the FEI TU Košice, Informatech, Ltd.,ISBN 80-88941-22-9, Košice

Jadlovska, A., Katalinic, B., Hrubina, K., Macurova,A., \& Wessely, E., (2011). Optimal Control of Nonlinear Systems with Constraint, Chapter 22, DAAAM International Scientific Book, In Katalinic, B.(Ed),Vienna, Austria, pp.265-282, ISBN 978-3-90150984-1, ISSN 1726-9687

Ljapunov,A.M. (1950). A General Task on the Motion Stability. Gosteizdat, Charkov, Malkin, I.G. (1966). The theory of the Motion Stability. Gostechizdat, Moskva, Poznyak, A. S. (2008). Avanced Mathematical Tools for Automatic Control Engineers. pp.774, Elsevier, ISBN 978080446 745, Amsterdam, New-York,

Tripathi, S.M. (2008). Modern Control Systemes (An Introduction), Infinity Science Pres LLC, ISBN 078-1-934015-21-6, Hingham, Massachusetts, New Delhi,

Zadech, L.A. (1973). A contribution to the theory of nonlinear systems, pp. 387-408 J. Franklin 